\title{
Introduction to Diophantine Approximation. Part II
}

\author{
Yasushige Watase \\ Suginami-ku Matsunoki 3-21-6 Tokyo \\ Japan
}

\begin{abstract}
Summary. In the article we present in the Mizar system [1, 2] the formalized proofs for Hurwitz' theorem [4, 1891] and Minkowski's theorem [5]. Both theorems are well explained as a basic result of the theory of Diophantine approximations appeared in [3], 6].

A formal proof of Dirichlet's theorem, namely an inequation $|\theta-y / x| \leqslant 1 / x^{2}$ has infinitely many integer solutions $(x, y)$ where $\theta$ is an irrational number, was given in [8]. A finer approximation is given by Hurwitz' theorem: $|\theta-y / x| \leqslant$ $1 / \sqrt{5} x^{2}$.
\end{abstract}

Minkowski's theorem concerns an inequation of a product of non-homogeneous binary linear forms such that $\left|a_{1} x+b_{1} y+c_{1}\right| \cdot\left|a_{2} x+b_{2} y+c_{2}\right| \leqslant \Delta / 4$ where $\Delta=\left|a_{1} b_{2}-a_{2} b_{1}\right| \neq 0$, has at least one integer solution.

\section{MSC: 11J20 11J25 03B35}

Keywords: Diophantine approximation; rational approximation; Dirichlet; Hurwitz; Minkowski

MML identifier: DIOPHAN2, version: 8.1.06 5.45.1311

\section{Preliminaries}

From now on $r_{1}, r_{2}, r_{3}$ denote non negative real numbers, $n, m_{1}$ denote natural numbers, $s$ denotes a real number, $i, j, i_{1}, j_{1}$ denote integers, $r$ denotes an irrational real number, and $q$ denotes a rational number.

Now we state the propositions:

(1) If $r_{1} \cdot r_{2} \leqslant r_{3}$, then $r_{1} \leqslant \sqrt{r_{3}}$ or $r_{2} \leqslant \sqrt{r_{3}}$.

(2) $\sqrt{r_{1} \cdot r_{2}}=\frac{r_{1}+r_{2}}{2}$ if and only if $r_{1}=r_{2}$. 
(3) $r_{1} \cdot r_{2}=\left(\frac{r_{1}+r_{2}}{2}\right)^{2}$ if and only if $r_{1}=r_{2}$. The theorem is a consequence of (2).

(4) If $i_{1}$ and $j_{1}$ are relatively prime, then there exist integers $s, t$ such that $s \cdot i_{1}+t \cdot j_{1}=1$.

(5) If $1<s$ and $s+\frac{1}{s}<\sqrt{5}$, then $s<\frac{\sqrt{5}+1}{2}$ and $\frac{1}{s}>\frac{\sqrt{5}-1}{2}$.

(6) If $q=\frac{i_{1}}{m_{1}}$ and $m_{1} \neq 0$ and $i_{1}$ and $m_{1}$ are relatively prime, then $i_{1}=\operatorname{num} q$ and $m_{1}=\operatorname{den} q$.

Let $f$ be a function. The functor ZeroPointSet $(f)$ yielding a set is defined by the term

(Def. 1) $\operatorname{dom} f \backslash \operatorname{support} f$. Now we state the proposition:

(7) Let us consider a function $f$, and objects $o_{1}$. Then $o_{1} \in \operatorname{ZeroPointSet}(f)$ if and only if $o_{1} \in \operatorname{dom} f$ and $f\left(o_{1}\right)=0$.

\section{Hurwitz' Theorem [4, 1891]}

Let $r$ be an irrational real number and $n$ be a natural number. Note that $(c d r)(n)$ is positive and natural. Now we state the propositions:

(8) Suppose $n>1$ and $\left|r-\frac{(c n r)(n)}{(c d r)(n)}\right| \geqslant \frac{1}{\sqrt{5} \cdot\left((c d r)(n)^{2}\right)}$ and $\left|r-\frac{(c n r)(n+1)}{(c d r)(n+1)}\right| \geqslant$ $\frac{1}{\sqrt{5} \cdot\left((c d r)(n+1)^{2}\right)}$. Then $\sqrt{5}>\frac{(c d r)(n+1)}{(c d r)(n)}+\frac{1}{\frac{(c d r)(n+1)}{(c d r)(n)}}$.

(9) If $i=(c n r)(n)$ and $j=(c d r)(n)$, then $i$ and $j$ are relatively prime.

(10) Suppose $n>1$. Then

(i) $\left|r-\frac{(c n r)(n)}{(c d r)(n)}\right|<\frac{1}{\sqrt{5} \cdot\left((c d r)(n)^{2}\right)}$, or

(ii) $\left|r-\frac{(c n r)(n+1)}{(c d r)(n+1)}\right|<\frac{1}{\sqrt{5} \cdot\left((c d r)(n+1)^{2}\right)}$, or

(iii) $\left|r-\frac{(c n r)(n+2)}{(c d r)(n+2)}\right|<\frac{1}{\sqrt{5} \cdot\left((c d r)(n+2)^{2}\right)}$.

The theorem is a consequence of (8) and (5).

Let us consider $r$. The functor $\operatorname{HWZSet}(r)$ yielding a subset of $\mathbb{Q}$ is defined by the term

(Def. 2) $\left\{p\right.$, where $p$ is a rational number $\left.:|r-p|<\frac{1}{\sqrt{5} \cdot\left((\operatorname{den} p)^{2}\right)}\right\}$.

The functor HWZSet1( $r$ ) yielding a subset of $\mathbb{N}$ is defined by the term

(Def. 3$) \quad\{x$, where $x$ is a natural number : there exists a rational number $p$ such that $p \in \operatorname{HWZSet}(r)$ and $x=\operatorname{den} p\}$.

The functor TRANQN yielding a function from $\mathbb{Q}$ into $\mathbb{N}$ is defined by

(Def. 4) for every rational number $x, i t(x)=\operatorname{den} x$.

(11) $(\operatorname{TRANQN})^{\circ}(\operatorname{HWZSet}(r))=\operatorname{HWZSet} 1(r)$. 
(12) If HWZSet $(r)$ is finite, then HWZSet1 $(r)$ is finite. The theorem is a consequence of (11).

Let us consider $r$. One can check that HWZSet1 $(r)$ is non empty.

(13) Let us consider a natural number $h$. If $h \in \operatorname{HWZSet1}(r)$, then $h>0$.

Let us consider $r$. Note that $\operatorname{HWZSet1}(r)$ is infinite.

(14) HuRWITZ'S THEOREM (NUMBER THEORY):

$\left\{q:|r-q|<\frac{1}{\sqrt{5} \cdot\left((\operatorname{den} q)^{2}\right)}\right\}$ is infinite. The theorem is a consequence of (12).

From now on $c_{0}, c_{1}, c_{2}, u, a_{0}, b_{0}$ denote real numbers.

Let $a_{0}, b_{0}, c_{0}$ be real numbers. The functor $\operatorname{LF}\left(a_{0}, b_{0}, c_{0}\right)$ yielding a function from $\mathbb{Z} \times \mathbb{Z}$ into $\mathbb{R}$ is defined by

(Def. 5) for every integers $x, y, i t(x, y)=a_{0} \cdot x+b_{0} \cdot y+c_{0}$.

\section{Minkowski's Theorem [5, Zweites Kapitel, §11, 1907]}

Now we state the proposition:

(15) Let us consider an element $\rho$ of $\mathbb{R}$, and integers $p, q$. Suppose $p$ and $q$ are relatively prime. Then there exist elements $x, y$ of $\mathbb{Z}$ such that $|p \cdot x-q \cdot y+\rho| \leqslant \frac{1}{2}$. The theorem is a consequence of (4).

In the sequel $a, b$ denote real numbers and $n$ denotes an integer.

(16) If $n \leqslant b \leqslant n+1$, then $|n-b| \cdot|n+1-b| \leqslant \frac{1}{4}$.

(17) If $a$ is not an integer and ( $n=\lfloor a\rfloor$ or $n=\lfloor a\rfloor+1)$, then $|a-n|<1$.

(18) Suppose $|n-a| \cdot|n+1-a| \leqslant \frac{1}{4}$ and $|n-b| \cdot|n+1-b| \leqslant \frac{1}{4}$. Then

(i) $|n-a| \cdot|n-b| \leqslant \frac{1}{4}$, or

(ii) $|n+1-a| \cdot|n+1-b| \leqslant \frac{1}{4}$.

The theorem is a consequence of (1).

(19) Suppose $|a-n| \cdot|b-n| \cdot|a-n-1| \cdot|b-n-1| \leqslant \frac{|a-b|^{2}}{4}$. Then

(i) $|a-n| \cdot|b-n| \leqslant \frac{|a-b|}{2}$, or

(ii) $|a-n-1| \cdot|b-n-1| \leqslant \frac{|a-b|}{2}$.

The theorem is a consequence of (1).

(20) Suppose $(n-b) \cdot(n+1-a)>0$ and $(a-n) \cdot(n+1-b)>0$. Then

(i) $(n-b) \cdot(n+1-a)+(a-n) \cdot(n+1-b)=a-b$, and

(ii) $|a-n| \cdot|b-n| \cdot|a-n-1| \cdot|b-n-1| \leqslant \frac{|a-b|^{2}}{4}$.

(21) If $b<n<a<n+1$, then $|a-n| \cdot|b-n| \cdot|a-n-1| \cdot|b-n-1| \leqslant \frac{|a-b|^{2}}{4}$. The theorem is a consequence of $(20)$. 
(22) Suppose $(n-a) \cdot(n+1-b)>0$ and $(b-n) \cdot(n+1-a)>0$. Then

(i) $(n-a) \cdot(n+1-b)+(b-n) \cdot(n+1-a)=b-a$, and

(ii) $|a-n| \cdot|b-n| \cdot|a-n-1| \cdot|b-n-1| \leqslant \frac{|a-b|^{2}}{4}$.

(23) If $n+1<b$ and $n<a<n+1$, then $|a-n| \cdot|b-n| \cdot|a-n-1| \cdot|b-n-1| \leqslant$ $\frac{|a-b|^{2}}{4}$. The theorem is a consequence of $(22)$.

(24) Suppose $a$ is not an integer and $\lfloor a\rfloor \leqslant b \leqslant\lfloor a\rfloor+1$. Then there exists an integer $u$ such that

(i) $|a-u|<1$, and

(ii) $|a-u| \cdot|b-u| \leqslant \frac{1}{4}$.

The theorem is a consequence of (16), (18), and (17).

(25) Suppose $|a-\lfloor a\rfloor| \cdot|b-\lfloor a\rfloor| \geqslant \frac{|a-b|}{2}$ and $|a-(\lfloor a\rfloor+1)| \cdot|b-(\lfloor a\rfloor+1)| \geqslant \frac{|a-b|}{2}$. Then

(i) $a$ is an integer, or

(ii) $\lfloor a\rfloor \leqslant b$.

The theorem is a consequence of (21), (19), and (3).

(26) Suppose $a$ is not an integer and $\lfloor a\rfloor>b$. Then there exists an integer $u$ such that

(i) $|a-u|<1$, and

(ii) $|a-u| \cdot|b-u|<\frac{|a-b|}{2}$.

The theorem is a consequence of (17) and (25).

(27) Suppose $|a-\lfloor a\rfloor| \cdot|b-\lfloor a\rfloor| \geqslant \frac{|a-b|}{2}$ and $|a-(\lfloor a\rfloor+1)| \cdot|b-(\lfloor a\rfloor+1)| \geqslant \frac{|a-b|}{2}$. Then

(i) $a$ is an integer, or

(ii) $\lfloor a\rfloor+1 \geqslant b$.

The theorem is a consequence of (23), (19), and (3).

(28) Suppose $a$ is not an integer and $\lfloor a\rfloor+1<b$. Then there exists an integer $u$ such that

(i) $|a-u|<1$, and

(ii) $|a-u| \cdot|b-u|<\frac{|a-b|}{2}$.

The theorem is a consequence of (17) and (27).

(29) There exists an integer $u$ such that

(i) $|a-u|<1$, and

(ii) $|a-u| \cdot|b-u| \leqslant \frac{1}{4}$ or $|a-u| \cdot|b-u|<\frac{|a-b|}{2}$. 
The theorem is a consequence of (24), (26), and (28).

In the sequel $a_{1}, a_{2}, b_{1}, b_{2}, c_{1}, c_{2}$ denote elements of $\mathbb{R}, \epsilon$ denotes a positive real number, $r_{1}$ denotes a non negative real number, and $q, q_{1}$ denote elements of $\mathbb{Q}$. Now we state the propositions:

(30) There exists an element $q$ of $\mathbb{Q}$ such that

(i) $\operatorname{den} q>\left\lfloor r_{1}\right\rfloor+1$, and

(ii) $q \in \operatorname{HWZSet}(r)$.

Proof: Reconsider $m=\left\lfloor r_{1}\right\rfloor+1$ as a natural number. There exists $n$ such that $n \in \operatorname{HWZSet} 1(r)$ and $n>m$ by (13), [7, (3)]. Consider $n$ such that $n \in \operatorname{HWZSet} 1(r)$ and $n>m$.

(31) Suppose $\left|a_{1} \cdot b_{2}-a_{2} \cdot b_{1}\right| \neq 0$ and $q \neq q_{1}$ and $a_{2} \cdot(\operatorname{den} q)+b_{2} \cdot(\operatorname{num} q)=0$. Then $a_{2} \cdot\left(\operatorname{den} q_{1}\right)+b_{2} \cdot\left(\operatorname{num} q_{1}\right) \neq 0$.

(32) Suppose $\left|a_{1} \cdot b_{2}-a_{2} \cdot b_{1}\right| \neq 0$. Then there exists an element $q$ of $\mathbb{Q}$ such that

(i) $\operatorname{den} q>\left\lfloor r_{1}\right\rfloor+1$, and

(ii) $q \in \operatorname{HWZSet}(r)$, and

(iii) $a_{2} \cdot(\operatorname{den} q)+b_{2} \cdot(\operatorname{num} q) \neq 0$.

The theorem is a consequence of (30) and (31).

(33) Let us consider real numbers $a_{1}, b_{1}$, and integers $n_{1}, d_{1}$. Suppose $d_{1}>0$

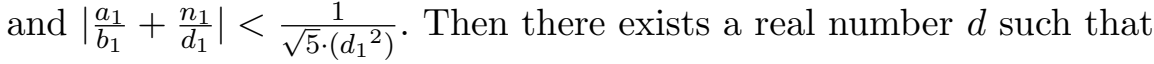

(i) $\frac{n_{1}}{d_{1}}=-\frac{a_{1}}{b_{1}}+\frac{d}{d_{1}^{2}}$, and

(ii) $|d|<\frac{1}{\sqrt{5}}$.

(34) Suppose $\left|a_{1} \cdot b_{2}-a_{2} \cdot b_{1}\right| \neq 0$ and $\frac{a_{1}}{b_{1}}$ is irrational. Then there exist elements $x, y$ of $\mathbb{Z}$ such that

(i) $\left|\left(\operatorname{LF}\left(a_{1}, b_{1}, c_{1}\right)\right)(x, y)\right| \cdot\left|\left(\operatorname{LF}\left(a_{2}, b_{2}, c_{2}\right)\right)(x, y)\right|<\frac{\left|a_{1} \cdot b_{2}-a_{2} \cdot b_{1}\right|}{4}$, and

(ii) $\left|\left(\operatorname{LF}\left(a_{1}, b_{1}, c_{1}\right)\right)(x, y)\right|<\epsilon$.

The theorem is a consequence of (32), (15), (29), and (33).

(35) Suppose $\left|a_{1} \cdot b_{2}-a_{2} \cdot b_{1}\right| \neq 0$ and $\frac{a_{2}}{b_{2}}$ is irrational. Then there exist elements $x, y$ of $\mathbb{Z}$ such that

(i) $\left|\left(\operatorname{LF}\left(a_{2}, b_{2}, c_{2}\right)\right)(x, y)\right| \cdot\left|\left(\operatorname{LF}\left(a_{1}, b_{1}, c_{1}\right)\right)(x, y)\right|<\frac{\left|a_{1} \cdot b_{2}-a_{2} \cdot b_{1}\right|}{4}$, and

(ii) $\left|\left(\operatorname{LF}\left(a_{2}, b_{2}, c_{2}\right)\right)(x, y)\right|<\epsilon$.

The theorem is a consequence of (34).

(36) Suppose ZeroPointSet $\left(\operatorname{LF}\left(a_{1}, b_{1}, c_{1}\right)\right) \neq \emptyset$. Then there exist elements $x, y$ of $\mathbb{Z}$ such that $\left|\left(\operatorname{LF}\left(a_{1}, b_{1}, c_{1}\right)\right)(x, y)\right| \cdot\left|\left(\operatorname{LF}\left(a_{2}, b_{2}, c_{2}\right)\right)(x, y)\right| \leqslant \frac{\left|a_{1} \cdot b_{2}-a_{2} \cdot b_{1}\right|}{4}$. The theorem is a consequence of $(7)$. 
(37) Suppose ZeroPointSet $\left(\operatorname{LF}\left(a_{2}, b_{2}, c_{2}\right)\right) \neq \emptyset$. Then there exist elements $x, y$ of $\mathbb{Z}$ such that $\left|\left(\operatorname{LF}\left(a_{1}, b_{1}, c_{1}\right)\right)(x, y)\right| \cdot\left|\left(\operatorname{LF}\left(a_{2}, b_{2}, c_{2}\right)\right)(x, y)\right| \leqslant \frac{\left|a_{1} \cdot b_{2}-a_{2} \cdot b_{1}\right|}{4}$. The theorem is a consequence of $(7)$.

(38) Suppose $\left|a_{1} \cdot b_{2}-a_{2} \cdot b_{1}\right| \neq 0$ and $b_{1} \neq 0$ and $\frac{a_{1}}{b_{1}}$ is rational. Then there exist elements $x, y$ of $\mathbb{Z}$ such that $\left|\left(\operatorname{LF}\left(a_{1}, b_{1}, c_{1}\right)\right)(x, y)\right| \cdot\left|\left(\operatorname{LF}\left(a_{2}, b_{2}, c_{2}\right)\right)(x, y)\right| \leqslant$ $\frac{\left|a_{1} \cdot b_{2}-a_{2} \cdot b_{1}\right|}{4}$. The theorem is a consequence of $(15)$.

(39) Suppose $\left|a_{1} \cdot b_{2}-a_{2} \cdot b_{1}\right| \neq 0$ and $b_{2} \neq 0$ and $\frac{a_{2}}{b_{2}}$ is rational. Then there exist elements $x, y$ of $\mathbb{Z}$ such that $\left|\left(\operatorname{LF}\left(a_{1}, b_{1}, c_{1}\right)\right)(x, y)\right| \cdot\left|\left(\operatorname{LF}\left(a_{2}, b_{2}, c_{2}\right)\right)(x, y)\right| \leqslant$ $\frac{\left|a_{1} \cdot b_{2}-a_{2} \cdot b_{1}\right|}{4}$. The theorem is a consequence of $(38)$.

(40) Suppose $\left|a_{1} \cdot b_{2}-a_{2} \cdot b_{1}\right| \neq 0$ and $b_{1}=0$. Then there exist elements $x, y$ of $\mathbb{Z}$ such that $\left|\left(\operatorname{LF}\left(a_{1}, b_{1}, c_{1}\right)\right)(x, y)\right| \cdot\left|\left(\operatorname{LF}\left(a_{2}, b_{2}, c_{2}\right)\right)(x, y)\right| \leqslant \frac{\left|a_{1} \cdot b_{2}-a_{2} \cdot b_{1}\right|}{4}$. The theorem is a consequence of (35), (37), and (39).

(41) Suppose $\left|a_{1} \cdot b_{2}-a_{2} \cdot b_{1}\right| \neq 0$. Then there exist elements $x, y$ of $\mathbb{Z}$ such that $\left|\left(\operatorname{LF}\left(a_{1}, b_{1}, c_{1}\right)\right)(x, y)\right| \cdot\left|\left(\operatorname{LF}\left(a_{2}, b_{2}, c_{2}\right)\right)(x, y)\right| \leqslant \frac{\left|a_{1} \cdot b_{2}-a_{2} \cdot b_{1}\right|}{4}$. The theorem is a consequence of (34), (36), (40), and (38).

\section{REFERENCES}

[1] Grzegorz Bancerek, Czesław Byliński, Adam Grabowski, Artur Korniłowicz, Roman Matuszewski, Adam Naumowicz, Karol Pąk, and Josef Urban. Mizar: State-of-the-art and beyond. In Manfred Kerber, Jacques Carette, Cezary Kaliszyk, Florian Rabe, and Volker Sorge, editors, Intelligent Computer Mathematics, volume 9150 of Lecture Notes in Computer Science, pages 261-279. Springer International Publishing, 2015. ISBN 978-3319-20614-1. doi 10.1007/978-3-319-20615-8_17.

[2] Adam Grabowski, Artur Korniłowicz, and Adam Naumowicz. Four decades of Mizar. Journal of Automated Reasoning, 55(3):191-198, 2015. doi 10.1007/s10817-015-9345-1

[3] G.H. Hardy and E.M. Wright. An Introduction to the Theory of Numbers. Oxford University Press, 6th edition, 2008.

[4] Adolf Hurwitz. Ueber die angenäherte Darstellung der Irrationalzahlen durch rationale Brüche Mathematısche Annalen, 39(2):279-284, B.G.Teubner Verlag, Leipzig, 1891.

[5] Hermann Minkowski. Diophantische Approximationen: eine Einführung in die Zahlentheorie. Teubner, Leipzig, 1907.

[6] Ivan Niven. Diophantine Approximation. Dover, 2008.

[7] Tetsuya Tsunetou, Grzegorz Bancerek, and Yatsuka Nakamura. Zero-based finite sequences Formalized Mathematics, 9(4):825-829, 2001.

[8] Yasushige Watase. Introduction to Diophantine approximation. Formalized Mathematics, 23(2):101-106, 2015. doi $10.1515 /$ forma-2015-0010.

Received November 29, 2017

The English version of this volume of Formalized Mathematics was financed under agreement 548/P-DUN/2016 with the funds from the Polish Minister of Science and Higher Education for the dissemination of science. 